\title{
Performance Investigation of Hydrostatic Transmission System as a Function of Pump Speed and Load Torque
}

\author{
S. K. Mandal $\cdot$ A. K. Singh $\cdot$ Y. Verma \\ K. Dasgupta
}

Received: 19 May 2008/Accepted: 8 April 2012/Published online: 2 June 2012

(C) The Author(s) 2012. This article is published with open access at Springerlink.com

\begin{abstract}
Every hydrostatic system requires a pump and a motor as its major components. Therefore, the operating parameters of the pump and the motor in a hydrostatic system are critical to the overall performance of the system. This paper evaluates the overall efficiency of the hydrostatic system based on the operating parameters of the pump and the motor, in this particular investigation they are the pump speed and the load torque, using the expressions obtained from the simple theory of Wilson (Hydraul Pneum Power 1:136-147, 1967). Many times in actual practice, various losses occurring in the hydrostatic system are not given proper consideration while the selection and design of the pump and the motor are made. The present article investigates the effects of the different loss coefficients of the pump and the motor on the overall performance of the system that may be useful for the selection of hydrostatic system and its components.
\end{abstract}

Keywords HST - Performance analysis - Open circuit . Loss coefficients $\cdot$ LSHT

\section{Abbreviations \\ $D_{\mathrm{m}} \quad$ Volumetric displacement rate of the motor \\ $D_{\mathrm{p}} \quad$ Volumetric displacement rate of the pump \\ $P_{1} \quad$ Suction pressure of the pump}

S. K. Mandal ( $\square)$, Member

Institute of Technology, Benaras Hindu University,

Varanasi 221 055, India

e-mail: mandalsantosh2000@yahoo.com

\author{
A. K. Singh, Non-member - Y. Verma, Non-member . \\ K. Dasgupta, Associate Member \\ Department of Mechanical Engineering and Mining Machinery \\ Engineering, Indian School of Mines, Dhanbad, India \\ e-mail: aloksingh_ism2004@hotmail.com
}

$P_{2} \quad$ Outlet pressure of the motor

$P_{1} \quad$ Pressure drop across the motor chamber

$P_{\mathrm{m}} \quad$ Inlet pressure of the motor

$P_{\mathrm{p}} \quad$ Discharge pressure of the pump

$P_{\mathrm{vm}} \quad$ Pressure drop across the valve port of the motor

$Q_{\mathrm{m}} \quad$ Outlet flow of the motor

$Q_{\text {mlkg }}$ Leakage flow of the motor

$Q_{\mathrm{p}} \quad$ Ideal supply flow rate of the pump

$Q_{\mathrm{plkg}} \quad$ Leakage flow of the pump

$Q_{\mathrm{s}} \quad$ Actual supply flow rate of the pump

$R_{\mathrm{ls}} \quad$ Loss coefficient of the pump

$R_{\text {mlkg }} \quad$ Leakage resistance of the motor

$R_{\text {plkg }} \quad$ Leakage resistance of the pump

$R_{\mathrm{vm}} \quad$ Valve port resistance

$T_{1} \quad$ Load torque of the motor

$T_{\mathrm{p}} \quad$ Input torque of the pump

$\omega_{\mathrm{m}} \quad$ Motor speed

$\omega_{\mathrm{p}} \quad$ Pump speed

$\eta \quad$ Overall efficiency of the hydrostatic transmission system

\section{Introduction}

Hydrostatic transmission (HST) system is one of the most important applications in the field of fluid power system. In the HST system, the mechanical energy of the input drive shaft of the pump is converted to pressure energy in a nearly incompressible working fluid and then reconverted into mechanical power from one source to another without the use of gears. HST can give smooth change of output speed, output torque and output power according to the design requirements. They are used to transmit rotating mechanical power from one source to another without use of gears in stepless manner. They are often used for speed 
control where large load-torque fluctuations may cause unacceptable changes in the output speed, also where the use of gear trains may be undesirable or impossible.

In an HST system, a pump, driven by a prime mover, supplies flow to drive a hydraulic motor, which in turn, drives a load connected to its shaft. If the displacement of the pump and motor are fixed, the HST system simply acts as a gearbox to transmit power from the prime mover to the load. The majority of HST systems, however, use a variable-displacement pump and a fixed displacement motor or both pump and motor may be of variable displacement type, so that speed, torque, or power can be suitably regulated.

The failure of HST systems frequently results from an inability to predict performance, rather than from failure of the transmission to perform as could be expected. The performance analysis of hydrostatic drive is important to establish the design guidelines based on the operating parameters of the major components of the system. From the accurately predicted performance of the HST system, the best combination of available components can be selected without building a series of prototype to obtain experimental data for comparison. The data regarding the characteristics of pump and motor, the major components of the system, are required to predict the overall performance of the HST system. The performance of the system depends on the operating parameters of the system such as pump speed, load torque and supply flow rate. The characteristics of various loss coefficients of pump and motor may be obtained experimentally. Using them for given load torque and pump speed, the efficiency of the hydrostatic drives can be predicted.

In due course of time, the leakages in the pump and the motor increase due to increase in the clearance spaces because of the wear and tear of the mechanical components. This, in turn, decreases their leakage resistances. The effects of variation of the loss coefficients on the overall performance of the system have been studied in this article.

Low speed high torque (LSHT) hydrostatic drives are widely used in heavy earth moving and construction equipment for their better control, smaller weight per unit power and compactness. It has good low speed characteristics without the need of a reduction gear unit and also has a high output torque throughout its range. The steady state performance investigation of the orbital rotor LSHT output hydraulic motor was carried out by Dasgupta et al. [1]. This article analyses the steady state performance of an open circuit LSHT hydrostatic drive that consists of a fixed displacement piston pump and an LSHT multi stroke radial piston motor. Different losses of the system have been lumped in the form of resistive elements. The effects of the various losses of the pump and the motor on the overall performance of the system have been studied. The expression relating to the efficiency of the system is derived based on the simple theory of Wilson [2].

\section{Physical System}

The simplified symbolic representation of an open circuit hydrostatic drive considered for the analysis is shown in Fig. 1. In an open circuit HST system, the flow path is not continuous and is interrupted by the oil reservoir. An electric motor drives a pressure compensated axial piston pump that supplies stabilized flow through direction control valve and pressure compensated flow control valve to the radial piston LSHT fixed displacement motor.

The system considered in the present investigation is an open circuit wherein the input power to the hydraulic pump and the output power from the hydraulic motor are separate entities and have no connection in between them. The
Fig. 1 The physical system (open circuit)

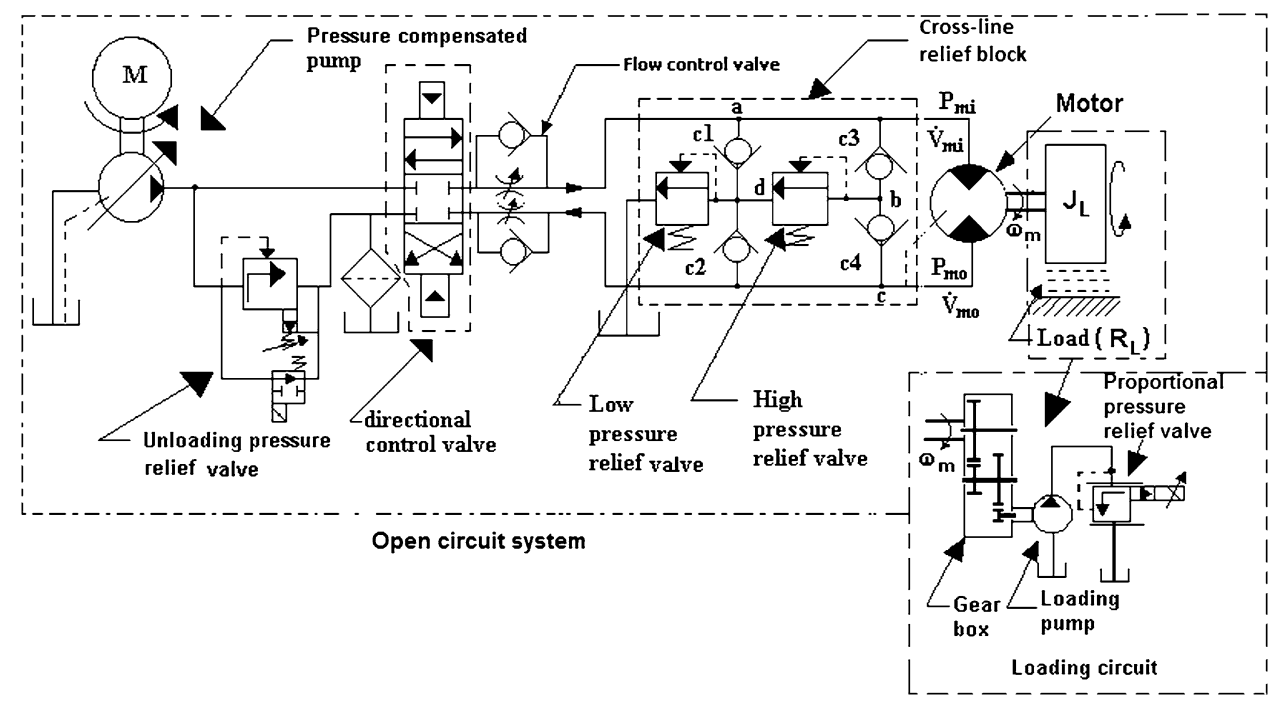


major components of the open circuit HST system considered in the present study are the pump and the motor. Using a variable frequency $(\mathrm{V} / \mathrm{F})$ controller, the speed of the electric motor and thereby the pump speed is varied. This, in turn, changes the flow supplied from the pump (flow is proportional to the pump speed) through a direction control valve and a flow control valve to the motor that drives the load connected to its shaft. The motor drives viscous and inertia loads. However, in experimental investigation of the system, the viscous load has been provided by a suitable loading arrangement. The inset picture shown in Fig. 1 indicates such an arrangement, where the motor drives a loading pump through a step up gear unit. By suitable adjustment of the proportional pressure relief valve connected to the outlet of the loading pump, the desired resistive load on the motor is achieved. Other essential parameters are sensed and recorded with the help of suitable sensors and instruments.

\section{Multi Stroke Radial Piston Motor}

Figure 2 shows the general constructional feature of a multi stroke radial piston hydrostatic motor [4]. The motor consists of two-part housing (1) and (2), rotary piston (3), output flange (6), control section (7), rotor assembly (4), roller bearing (8), cam plate (5), inlet port (A), outlet port (B), drain port (L) and annular passages (D). There are eight pistons, which are radially placed with equal angular spaced in the rotor and is supported on the cam plate by way of rollers. The rotor is connected to the output shaft flange by means of a spline. The output flange is mounted on the shaft over a set of taper roller bearings. Similarly, the cylinder assembly is also kept over a set of taper roller bearings. The two parts of the motor were fastened with the help of bolts. The annular passages (D) are connected with the cylinder inlet and outlet ports by way of axial holes. A case drain port (L) is provided to accommodate internal leakage.
The cylinder chamber is supplied with fluid via the axial bores in the control. Each piston is charged with fluid as many times per revolution of the shaft as there are number of cams on the stroke cam. The torque resulting from the curve of the cam is transferred from the piston group to the output shaft via a spline. A tapered roller bearing is integrated into the housing which is capable of receiving high axial and radial forces.

The motor housing comprises of two channels, one for fluid inlet and one for fluid outlet. Both the channels are connected with all the cylinder chambers in such a way that for different position of pistons one or the other channel opens. When liquid is forced into the cylinder bore containing piston I from the inlet channel, the piston I moves outward since the liquid cannot be compressed. As the force acting on piston I cause the cylinder block to rotate, piston II starts to rotate and approach the position of piston III. As piston II rotates, it is forced inward and, in turn, forces the fluid out of the cylinder through the outlet channel to the sump. The same cycle is followed for the rest part of the rotation of the shaft. Depending upon the position of the pistons, the different cylinder chambers are connected to the inlet and outlet port. Due to the rotation of the rotor, torque is produced. The direction of rotation of the motor can be changed by reversing the flow of fluid to it.

There exist leakages between the piston and the cylinder wall through the small clearance between the two and the cross-port leakage between the high pressure ports and the low pressure ports of the motor. Together, these leakages are termed as internal leakage of the motor and its leakage resistance is denoted by $R_{\mathrm{mlkg}}$. Similar leakages are present in the pump and are termed as internal leakage of the pump and its leakage resistance is denoted by $R_{\mathrm{plkg}}$. As the flow supplied from the pump enters the cylinder chamber of the motor, there is a pressure drop across the valve port due to the resistance at the valve port. This valve port resistance is denoted by $R_{\mathrm{vm}}$. In the present analysis, depending on the pump speed, the stable source of flow is considered to be
Fig. 2 Multi stroke radial piston motor
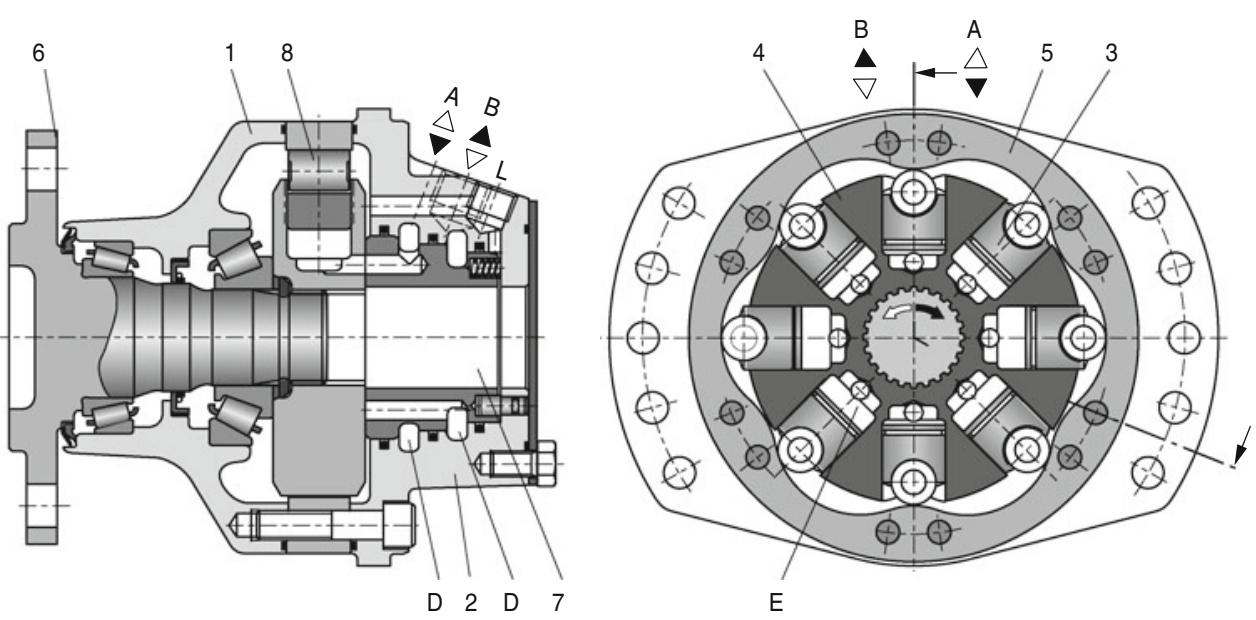
supplied from flow control valve to the inlet port of the motor. Thereby, the resistances of the direction control valve and the flow control valve as shown in Fig. 1 are not considered for simplification of the analysis.

\section{Theoretical Considerations}

Referring to discussions made in "Multi Stroke Radial Piston Motor" section, the simplified schematic representation of the physical system considered for the analysis is shown in Fig. 3. The induction motor (M) drives the hydraulic pump that supplies flow to the hydraulic motor, which in turn, drives the load.

As mentioned earlier, the hydraulic pump and the motor are of fixed displacement types. There are internal leakages both in the pump and the motor. The leakages in positive displacement pumps and motors are primarily a result of the flow through the small clearance spaces between the various members that separate high-pressure regions from low-pressure ones. In a piston pump and the motor, mainly the leakage is between the piston and the cylinder wall. Additional leakage paths may connect the high-pressure ports to its low-pressure ports within the pump and the motor. The small spaces that permit leakage in the pump and the motor are often referred to as capillary passages. Most of the leakage paths are essentially between two flat plates with the flow occurring through the clearance spaces. The leakage flow is laminar, which is described by the fundamental relationship of the flow between flat plates. The leakage flow of pump and motor are given by

$Q_{\mathrm{plkg}}=\frac{P_{p}-P_{1}}{R_{\mathrm{plkg}}}$

$Q_{\mathrm{mlkg}}=\frac{P_{l}-P_{2}}{R_{\mathrm{mlkg}}}$

where $P_{\mathrm{p}}-P_{1}=$ pressure across the pump; $P_{1}-$ $P_{2}=$ pressure across the motor; $Q_{\mathrm{plkg}}=$ leakage flow of the pump; $Q_{\mathrm{mlkg}}=$ leakage flow of the motor; $R_{\text {plkg }}=$ leakage resistance of the pump; $R_{\mathrm{mlkg}}=$ leakage resistance of the motor.

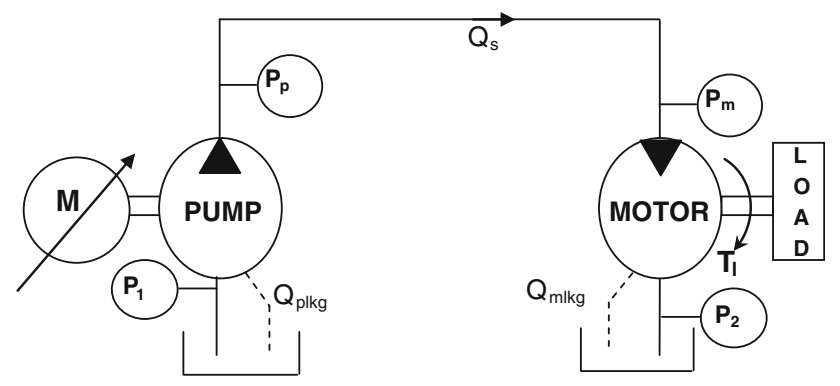

Fig. 3 Hydrostatic transmission system
The pump and the motor leakages increase the slip of the hydrostatic drive and hence decreases the overall efficiency of the system.

The load torque of the motor is given by

$T_{1}=\left(P_{1}-P_{2}\right) D_{\mathrm{m}}$

where $D_{\mathrm{m}}=$ volumetric displacement rate of motor.

Now, considering the mechanical losses of the pump,

Input torque of the pump,

$T_{\mathrm{p}}=\left(P_{\mathrm{p}}-P_{1}\right) D_{\mathrm{p}}+R_{\mathrm{ls}} \omega_{\mathrm{p}}$

where $R_{\mathrm{ls}}=$ mechanical loss coefficient of the pump; $D_{\mathrm{p}}=$ volumetric displacement rate of the pump; $\omega_{\mathrm{p}}=$ pump speed.

Based on the continuity equation of the fluid flow and neglecting the leakage loss in the line connecting the pump and the motor, the flow equation for the hydrostatic transmission is given by, actual supply flow rate of the pump $=$ inlet flow of the motor.

Hence,

$Q_{\mathrm{p}}-Q_{\mathrm{plkg}}=Q_{\mathrm{s}}$

$\omega_{\mathrm{p}} D_{\mathrm{p}}-\frac{P_{\mathrm{p}}-P_{1}}{R_{\mathrm{plkg}}}=Q_{\mathrm{s}}$

where $Q_{\mathrm{p}}=$ ideal supply flow rate of the pump; $Q_{\mathrm{s}}=$ actual supply flow rate of the pump.

Now, considering the leakage flow of the motor

$Q_{\mathrm{s}}=Q_{\mathrm{mlkg}}+Q_{\mathrm{m}}$

$Q_{\mathrm{s}}=\frac{P_{1}-P_{2}}{R_{\mathrm{mlkg}}}+\omega_{\mathrm{m}} D_{\mathrm{m}}$

where $Q_{\mathrm{m}}=$ Outlet flow of the motor.

From Eqs. 3 and 4,

$\omega_{\mathrm{p}} D_{\mathrm{p}}-\frac{P_{\mathrm{p}}-P_{1}}{R_{\mathrm{plkg}}}=\omega_{\mathrm{m}} D_{\mathrm{m}}+\frac{P_{1}-P_{2}}{R_{\mathrm{mlkg}}}$

Referring to the characteristic features of the radial piston motor described in "Multi Stroke Radial Piston Motor" section, the flow enters into the piston chamber through the inlet valve ports and comes out from the motor through its outlet valve ports. Due to the load torque $T_{1}$, the load pressure $P_{1}$ exists across the high-pressure and the low-pressure chamber of the motor. As the flow enters into the motor chamber through the valve ports, there is a loss of pressure across the ports. This pressure loss mainly contributes to the loss of mechanical efficiency of the motor and hence the overall efficiency of the system. The flow entering into the motor chamber through the valve port is expressed as

$\frac{P_{\mathrm{m}}-P_{1}}{R_{\mathrm{vm}}}=\omega_{\mathrm{m}} D_{\mathrm{m}}+\frac{P_{1}-P_{2}}{R_{\mathrm{mlkg}}}$

In the above equation, $\omega_{m} D_{m}$ is the outlet flow of the motor, $\frac{P_{1}-P_{2}}{R_{\mathrm{mkg}}}$ is the leakage flow of the motor, where $P_{\mathrm{m}}$ is 
the inlet pressure of the motor, $P_{1}$ is the pressure drop across the motor chamber.

Although the above equations require a certain pressure difference across the motor and the pump, there are infinite numbers of individual pressures which can have the same difference. Therefore the final bit of information is obtained by specifying $P_{\mathrm{p}}, P_{1}, P_{1}$ or $P_{2}$ or by obtaining a relation between these quantities as there is more number of variables than the number of equations. Considering $P_{1}$ and $P_{2}$ equal to zero and neglecting the resistance in the line between the pump and the motor,

$P_{\mathrm{m}}=P_{\mathrm{p}}$

The load torque produced by the motor is given by

$T_{1}=P_{1} D_{\mathrm{m}}$

where $P_{1}=$ pressure drop across the motor chamber.

Considering the mechanical losses of the pump, the input torque of the pump is given by

$T_{\mathrm{p}}=P_{\mathrm{p}} D_{\mathrm{p}}+R_{\mathrm{ls}} \omega_{\mathrm{p}}$

where $P_{\mathrm{p}}=$ discharge pressure of the pump; $R_{\mathrm{ls}}=$ mechanical loss coefficient of the pump.

The flow equation for the hydrostatic transmission is given by

$\omega_{\mathrm{m}} D_{\mathrm{m}}=D_{\mathrm{p}} \omega_{\mathrm{p}}-\frac{P_{\mathrm{p}}}{R_{\mathrm{plkg}}}-\frac{P_{1}}{R_{\mathrm{mlkg}}}$

And the flow supplied to the piston motor chamber is given by

$\frac{P_{\mathrm{p}}-P_{1}}{R_{\mathrm{vm}}}=\omega_{\mathrm{m}} D_{\mathrm{m}}+\frac{P_{1}}{R_{\mathrm{mlkg}}}$

Considering one of the piston cylinder assemblies, the pressure outside the valve port is more than the pressure inside the cylinder. This is due to the fact that there is a pressure drop across the valve port. This pressure drop is expressed by

$P_{\mathrm{vm}}=Q_{\mathrm{s}} R_{\mathrm{vm}}$

where $R_{\mathrm{vm}}=$ valve port resistance.

The characteristics of the valve port resistance are expressed by the following empirical relation which was established by the experimental investigation; the details of which is not discussed here.

$$
\begin{aligned}
R_{\mathrm{vm}} \times 10^{-9}= & \left(-0.0007 T_{1}+0.5509\right) D_{\mathrm{p}} \omega_{\mathrm{p}} \\
& +\left(0.0125 T_{1}+1.2874\right)
\end{aligned}
$$

where $T_{1}=$ load torque of the motor; $R_{\mathrm{vm}}=$ valve port resistance

The overall efficiency of the system is given by

$\eta=\frac{T_{1} \omega_{\mathrm{m}}}{T_{\mathrm{p}} \omega_{\mathrm{p}}}$
Solving the above six equations i.e. Eqs. 7, 8, 9, 10, 11 and 12 , the efficiency in terms of load torque $\left(T_{1}\right)$ and pump speed $\left(\omega_{\mathrm{p}}\right)$ is given by,

$\eta=\frac{T_{1}\left[D_{\mathrm{p}} \omega_{\mathrm{p}}-\frac{T_{\mathrm{l}}}{D_{\mathrm{m}} R_{\mathrm{mlkg}} R_{\mathrm{plkg}}}\left[R_{\mathrm{plkg}}+R_{\mathrm{mlkg}}+R_{\mathrm{vm}}\right]\right]}{\omega_{\mathrm{p}}\left[T_{1} D_{\mathrm{p}}+R_{\mathrm{ls}} \omega_{\mathrm{p}} D_{\mathrm{m}}+D_{\mathrm{m}} \omega_{\mathrm{p}} R_{\mathrm{vm}}\left(D_{\mathrm{p}}^{2}+\frac{R_{\mathrm{l}}}{R_{\mathrm{plkg}}}\right)\right]}$.

\section{Results and Discussions}

As seen in the Eq. 13, the overall efficiency of the HST system depends on pump speed $\omega_{\mathrm{p}}$ and load torque $T_{1}$ with the consideration that the pump leakage resistance $R_{\mathrm{plkg}}$, motor leakage resistance $R_{\mathrm{mlkg}}$, and mechanical loss coefficient $R_{\mathrm{ls}}$ are constant. Following the standard procedures [3], the hydrostatic drive shown in Fig. 1 is tested at various drive speed of the pump $\omega_{\mathrm{p}}$ and the load torque $T_{1}$, which are measured by suitable sensors and instruments. Similarly, the geometrical parameters of the pump $\left(D_{\mathrm{p}}\right)$ and the motor $\left(D_{\mathrm{m}}\right)$ are obtained from their product catalogues [4] and [5]. The values of the loss coefficients of the pump and the motor i.e. $R_{\mathrm{plkg}}, R_{\mathrm{mlkg}}, R_{\mathrm{ls}}$ are estimated from their volumetric and mechanical efficiencies. Using these parameters, the characteristics of the overall efficiency of the system with respect to the pump speeds at different load torque levels are plotted in Fig. 4. The effects of the variation of the loss coefficients on the overall steady state performance of the system at various torque levels are shown in Figs. 5, 6 and 7.

In evaluating the performance of the HST system, the pump speed is varied from $50 \mathrm{rad} / \mathrm{s}(600 \mathrm{rpm})$ to $150 \mathrm{rad} / \mathrm{s}$ $(1,450 \mathrm{rpm})$. Due to the irregular flow characteristics of the pump below $600 \mathrm{rpm}$, the test is carried out for the pump speed above $600 \mathrm{rpm}$. The pump is driven by a 4-pole

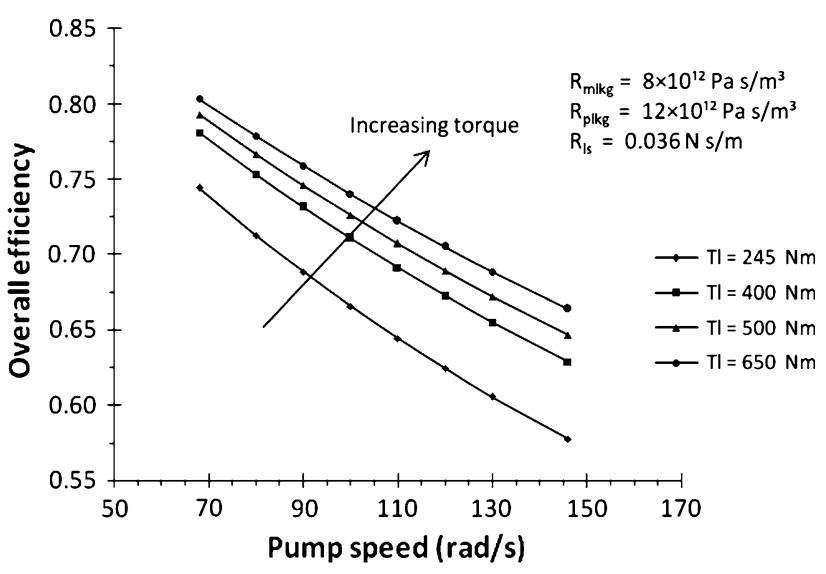

Fig. 4 Overall efficiency versus pump speed 


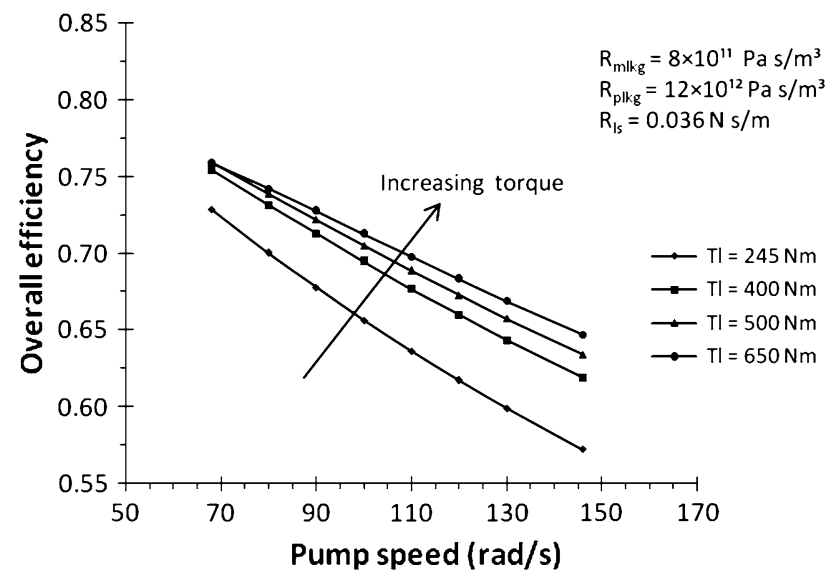

Fig. 5 Overall efficiency versus pump speed

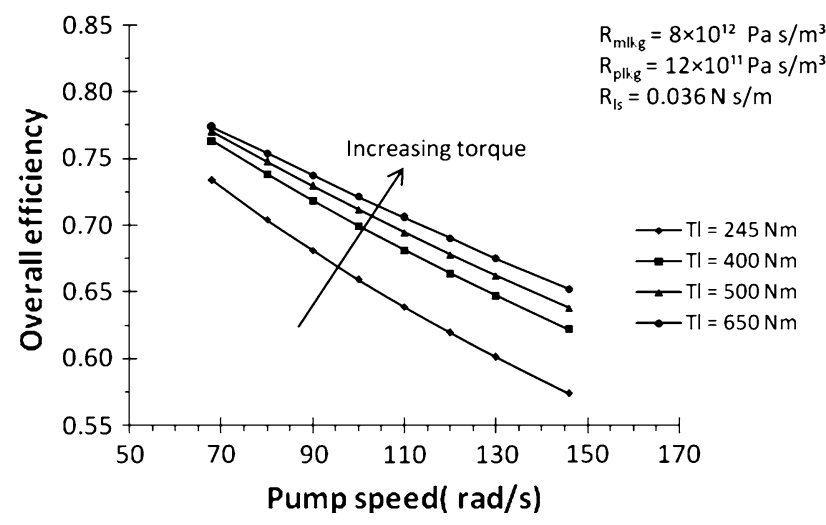

Fig. 6 Overall efficiency versus pump speed

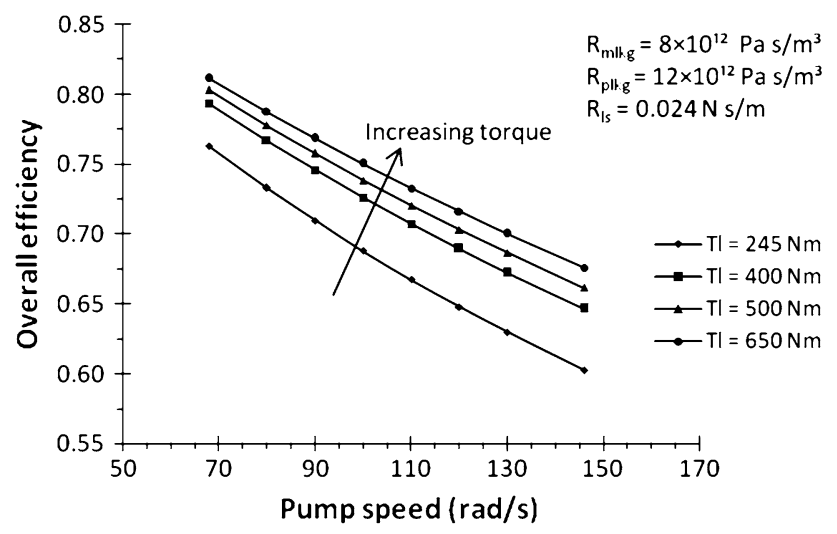

Fig. 7 Overall efficiency versus pump speed

induction motor, the maximum speed of which is limited to $1,450 \mathrm{rpm}$.

Figures 4 and 5 compare the effect of the decrease of $R_{\mathrm{mlkg}}$ on the overall efficiency of the system. Keeping the other loss coefficients constant, the ten-fold decrease in the value of $R_{\mathrm{mlkg}}$ decreases the overall efficiency of the system by $1.0-5.4 \%$.
Figures 4 and 6 give the relative analysis of the effect of the decrease of $R_{\text {plkg }}$ on the overall efficiency of the system. Keeping the other loss coefficients constant, the tenfold decrease in the value of $R_{\text {plkg }}$ decreases the overall efficiency of the system by $0.7-3.7 \%$.

Figures 4 and 7 account for the comparative analysis of the effect of decrease of $R_{\mathrm{ls}}$ on the overall efficiency of the system. Keeping the other loss coefficients constant, the two-third decrease in the value of $R_{\mathrm{ls}}$ increases the overall efficiency of the system by $1.0-4.3 \%$.

As can be seen from the above figures i.e. Figs. 4, 5, 6 and 7 that for a given load torque $\left(T_{1}\right)$ the overall efficiency of the system decreases uniformly with the increase in the pump speed $\left(\omega_{\mathrm{p}}\right)$. It is also observed that at a particular $\omega_{\mathrm{p}}$, the overall efficiency of the system increases with the increase in $T_{1}$. The decrease in efficiency at higher value of $\omega_{\mathrm{p}}$ and lower value of $T_{1}$ is due to the increase in value of $R_{\mathrm{vm}}$ as is evident from Eq. 11. However, at higher load torque, the leakage losses of the pump and the motor are more predominant. Therefore, the hydrostatic drives considered here are more suitable for high torque and low speed applications.

\section{Conclusions}

Experience has revealed that the overall performance of the HST system was based on the output parameters only i.e. the motor speed and its torque. However, this was not adequate as the performance depended on many other factors as well. This is revealed by the fact that the performance of the HST system at one set of output parameters was not constant and changed with variation in other parameters than the set of output parameters. To investigate this phenomenon, this article analyzed the overall performance of an open circuit hydrostatic drive as a function of pump speed and load torque taking into account the various loss coefficients of the components of the system. The analysis has been carried out to investigate the effects of the operating parameters of the pump and the motor on the overall efficiency of the system. Based on the analysis, the following conclusions were deduced:

- The loss coefficients of the pump and the motor affect the overall performance of the system and hence should be taken into account while selection and design of the hydrostatic drive are made.

- The operating parameters of the pump and the motor i.e. the pump speed and the load torque have significant impact on the overall performance of the HST system and should be taken care of while the system is in operation. 
- The wear and tear of the mechanical components results in the increase in the clearance spaces. This causes increase in the leakages and decrease in the leakage resistances which affects the overall performance of the hydrostatic drive. Thus suitable procedures should be taken up to minimize the wear and tear of the components while they are in operation.

Based on the analysis, it was found that the hydrostatic drive considered in the present study is suitable for high torque and low speed applications.

The leakage resistances of the pump and the motor $R_{\mathrm{plkg}}$ and $R_{\mathrm{mlkg}}$ mainly depend on the clearances between the mating parts. These clearances may vary with the load and the speed of the motor and the hydrodynamic lubrication, which are very difficult to measure under running conditions. Considering these effects, further refinement of the model may be made. The authors believe that the studies conducted here may be useful to the practicing engineers and it may also be helpful for the selection of similar machines.
Open Access This article is distributed under the terms of the Creative Commons Attribution License which permits any use, distribution, and reproduction in any medium, provided the original author(s) and the source are credited.

\section{References}

1. K. Dasgupta, A. Mukherjee, R. Maiti, Analytical and experimental studies of the steady-state performance of an orbital rotor lowspeed high-torque hydraulic motor. J. Power Energy A 210 , 423-429 (1996)

2. W.E. Wilson, Mathematical models in fluid power engineering. Hydraul. Pneum Power 1, 136-147 (1967)

3. British Standard Institution, Methods of testing hydraulic pumps and motors for hydraulic power transmission (British Standard Institution, London, 1970)

4. Bosch Rexroth India Ltd, Product Catalogue: RE 15 205/05.93, Hydraulic Motor MCR3F280F 180Z32B2M Technical Information (Bosch Rexroth India Ltd, Vatva, 2012)

5. Bosch Rexroth India Ltd, Product Catalogue: RE 92711/03.93, Hydraulic Pump (Pressure Compensated Fixed Displacement Type) A10VSO28DR/3X RPPA 12N00, Technical Information (Bosch Rexroth India Ltd, Vatva, 2012) 